\title{
RFI in Metsähovi Radio Observatory - measurements and effects
}

\section{Petri Kirves*,}

Aalto University Metsähovi Radio Observatory

Metsähovintie 114, FIN-02540 Kylmälä, Finland

E-mail: pkirves@kurp.hut.fi

\section{Juha Kallunki}

E-mail: kallunki@kurp.hut.fi

\section{Jan Wagner}

E-mail: jwagner@kurp.hut.fi

\begin{abstract}
Metsähovi Radio Observatory has over 12 years history of monitoring radio frequency interference (RFI). The system makes it possible to detect normal radio frequency signals (TV-channels, cell phones), which are harmful for radio astronomy observations. RFI monitoring frequency range corresponds to the IF frequency range of the receivers. In order to keep the downconverted signal clean of interference, it is essential to maintain good shielding of the IF cables. Clearly noticeable interference pulses are seldom found on beam difference monitoring system plots, but when found the RFI measurement system helps to trace down the reasons of the events.

RFI mitigation is realized by avoiding any unnecessary noise sources, cell phone usage, fluorescent lamps, wireless LANs etc.
\end{abstract}

RFI mitigation workshop - RFI2010,

March 29-31, 2010

Groningen, the Netherlands

\footnotetext{
* Speaker.
} 


\section{General}

Metsähovi Radio Observatory is situated in southern Finland, some $45 \mathrm{~km}$ from the capital, Helsinki. The nearest village is around $10 \mathrm{~km}$ away and the nearest settlements reside at a $550 \mathrm{~m}$ distance. The observatory area with a radius of $7 \mathrm{~km}$ has some special frequency usage restrictions in force. The area can be described as a semi-remote location for radio astronomy observations.

RFI monitoring has been performed in Metsähovi Radio Observatory (MRO) since 1998. The idea is to observe long-term variations and changes in RFI in the IF band (400 - $2000 \mathrm{MHz})$ of the receivers [1]. At the time of system construction, mobile communication was growing fast. This motivated us to monitor the possible impact on MRO observations. The observation bands at MRO (listed in table 1) are on centimeter and millimeter bands which, at least until now, makes it technically challenging and expensive to realize a RFI measurement system directly in the RF band.

Table 1: MRO obs bands

\begin{tabular}{|c|c|c|c|}
\hline band & MRO frequency [GHz] & IF [GHz] & other users \\
\hline \hline S & $2.21-2.35$ & $0.68-0.82$ & links, wireless cameras \\
\hline X & $8.15-8.65$ & $0.50-0.98$ & links, digital radiolink systems \\
\hline K & $21.0-22.0,22.4-23.4$ & $0.2-1.2$ & sat down, short range devices, links \\
\hline K & $21.98-22.48$ & $0.5-1.0$ & short range devices, links \\
\hline Q & $35.3-36.3,37.3-38.3$ & $0.5-1.5$ & radar, digital radiolink systems, sat down \\
\hline W & $86-98$ & $3.5-4.5,0.5-1.0$ & navigation \\
\hline
\end{tabular}

\section{Measurements}

A simplified overview showing the technical construction of the MRO RFI measurement system is presented in figure 1 . RFI signals are received with a log-periodic antenna with $+6 \mathrm{dBi}$ gain. The antenna is located on the roof of the laboratory building, on top of a separate antenna tower at a height of 4 meters from ground level. The antenna has a rotation motor so the horizontal pointing can be controlled freely. However, only four different antenna positions are actually in use. The received signal is amplified with a separate pre-amplifier with a gain of around $+30 \mathrm{~dB}$ and is then transmitted to the laboratory building. In the laboratory a portable spectrum analyzer detects the signal. Two different computers are needed to control the spectrum analyzer (data collection + control commands) and the antenna rotation motor. Our spectrum analyzer can be controlled via Ethernet (Telnet protocol).

The most powerful signals are TV channels and cell phone links, GSM-900 and GSM-1800 and the former analog NMT-450. Comparing graphs from different years reveals changes in overall spectrum usage and thus the level of possible RFI. The transition from analog to digital TV broadcasting is clearly visible due to frequency and modulation envelope differences. The main observed RFI signals are listed in table 2 .

The RFI measurement system covers the IF bandwidth (400 - $2000 \mathrm{MHz})$. Because of limited resolution and sweep time duration, the IF band is divided into $100 \mathrm{MHz}$ bands. Each of these 


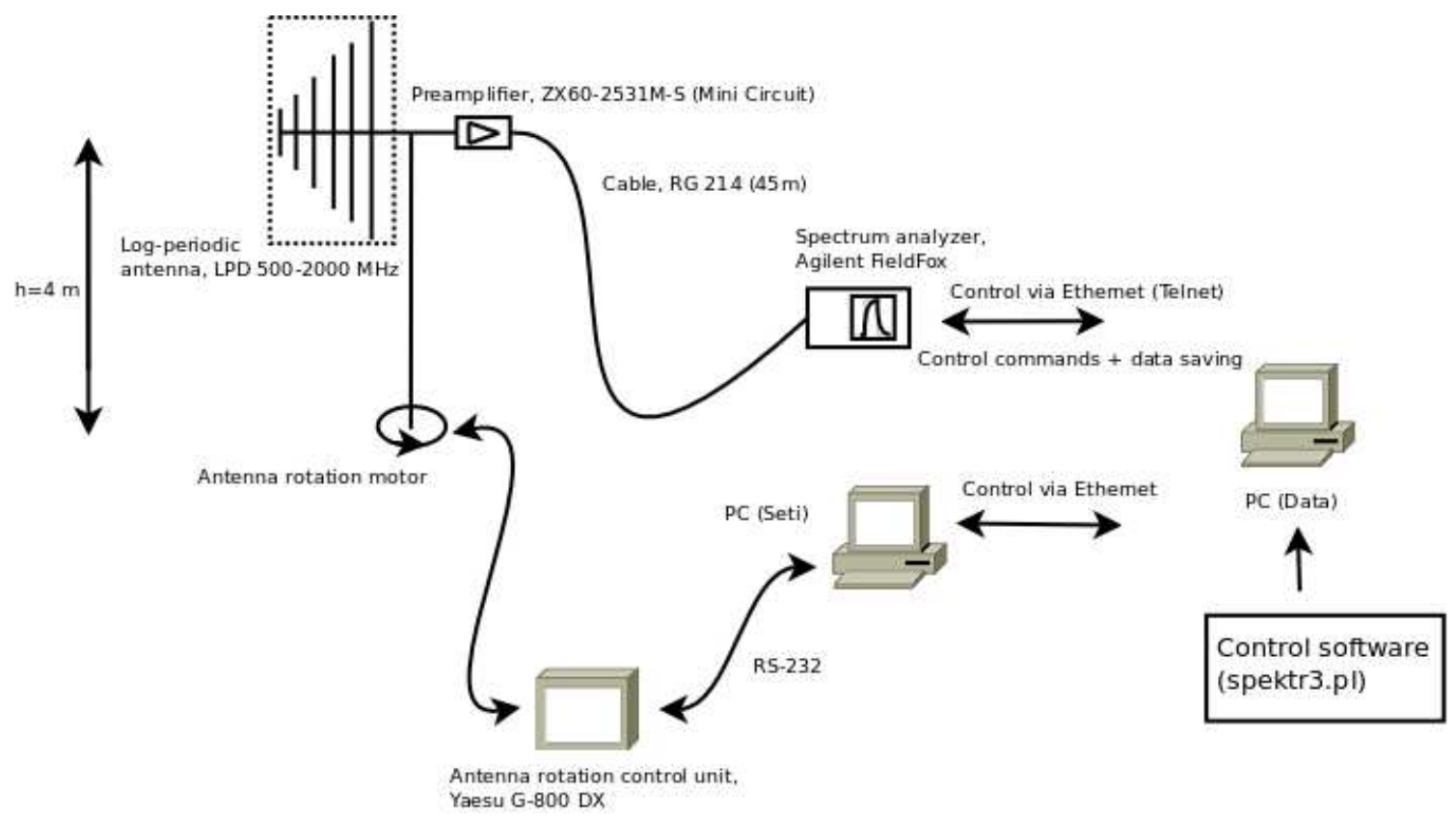

Figure 1: Overview of the RFI measuring system at MRO.

Table 2: main observed RFI

\begin{tabular}{|c|c|c|}
\hline ID & $\mathrm{f}[\mathrm{MHz}]$ & description \\
\hline \hline analog TV ch 24 & 498 & TV3 \\
\hline analog TV ch 35 & 586 & SVT \\
\hline analog TV ch 46 & 674 & TV1 sub \\
\hline analog TV ch 52 & 722 & TV4 \\
\hline digital TV ch 32 & 562 & multiplex A \\
\hline digital TV ch 44 & 658 & multiplex B \\
\hline digital TV ch 46 & 674 & multiplex E \\
\hline digital TV ch 53 & 730 & downlink 463 - 468, uplink 453 - 458 \\
\hline NMT 450 & 458 & downlink 921 - 960, uplink 876 - 915 \\
\hline GSM 900 & 900 & 1805 - 1880, uplink 1710 - 1785 \\
\hline GSM 1800 & 1800 & downlink 1805 \\
\hline
\end{tabular}

sixteen $100 \mathrm{MHz}$ band is scanned ten times with the spectrum analyzer's max hold option. As mentioned previously, four different antenna positions are used - the principal compass points. Thus 64 band scans will be performed in total. Using a $100 \mathrm{MHz}$ width, a resolution bandwidth of $3 \mathrm{kHz}$ and video bandwidth of $1 \mathrm{kHz}$, the sweep time for one scan is 6,1 seconds. 1000 points are collected on each sweep. Completing the 64 scans takes about 80 minutes.

The latest addition made to the system was to extend the scanned frequencies into S-band (2,21 - 2,35 GHz). Several other users near the observation band can be identified, such as links, amateur radio and microwave ovens. There is also intermittent RFI in the observation band that can be seen in the 24-day accumulated data. Currently when interference appears in narrow band observations, 
a rare event, the problem is circumvented by simply changing the observing frequency a little.

The susceptibility of the receiver electronics to external interference was tested using a simple method. We launched a GSM call and moved the transmitting phone all over the enclosure. Of course this kind of event is not expected in a real observation. Nevertheless it acts as a fingerprint of how a strong, pulse shape interference looks like in the measurement data (figure 2). It was learned that interference near the receiver installation platform can couple in, but in the control room the signal path seems to be shielded well enough.

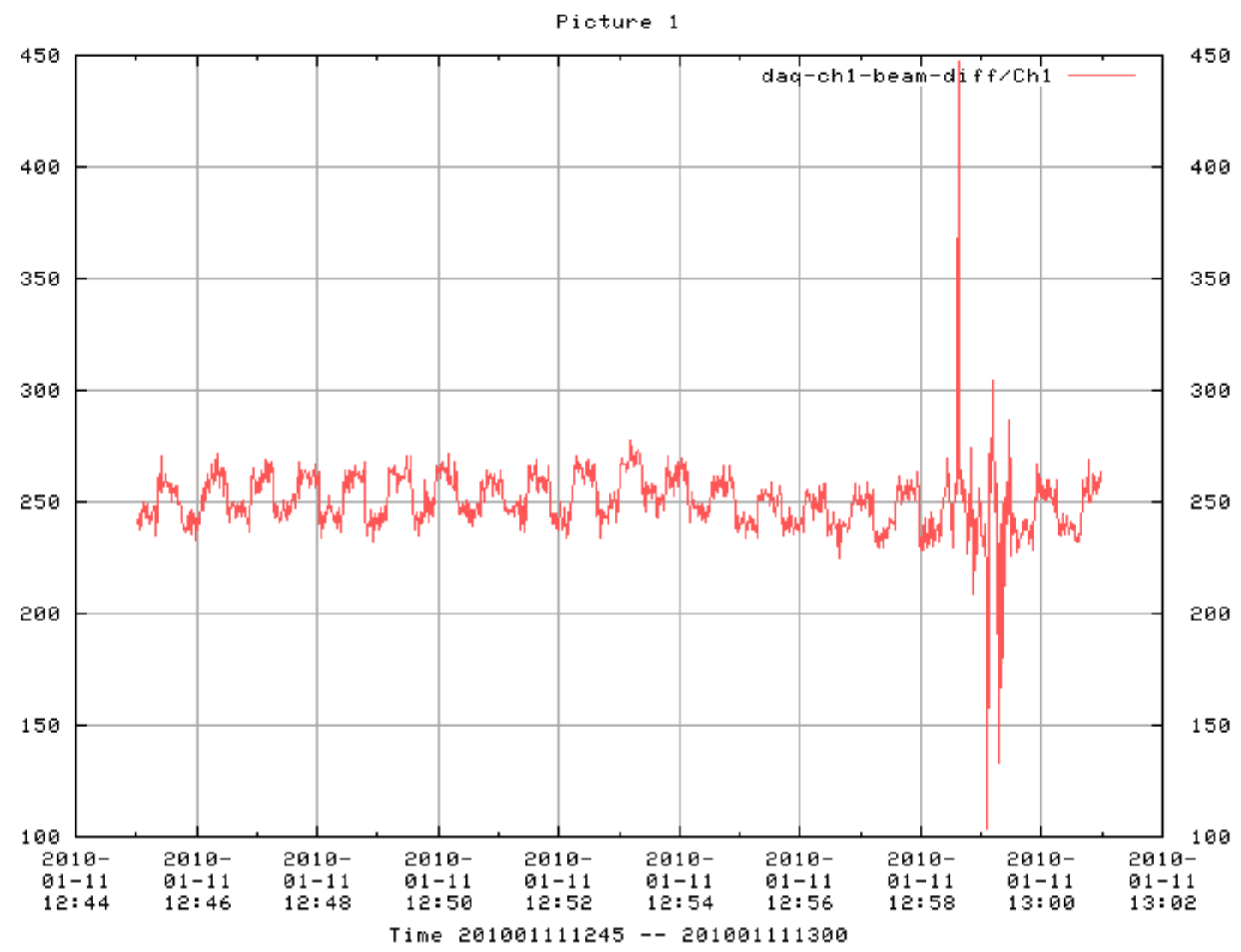

Figure 2: GSM interference signal in the beam difference data. Vertical scale is the detected signal level in arbitrary units.

\section{RFI Detection in Software}

Before and during the EUR103 geodetic VLBI session in the winter of 2010, we prepared one of our Berkeley CASPER iBOB FPGA-boards to monitor the S-band IF, sampled with one iADC $2 \times 1 \mathrm{Gs} / \mathrm{s}$ A/D converter board [2]. The IF was band-pass filtered for $512-1000 \mathrm{MHz}$ to work the A/D in undersampling mode. The FPGA was programmed with the Kurtosis Spectrometer developed for the Korea Solar Radio Burst Locator [3]. A modified NI LabView program was used to capture the iBOB data from $100 \mathrm{Mbps}$ LAN and record the results of online RFI detection onto disk. 
The Korean firmware samples the IF at a $1 \mathrm{Gs} / \mathrm{s}$ sampling rate and computes real-time PSD estimates with 2048 channels (DC to Nyquist) and spectral power squared values. They are accumulated over a configurable integration time interval ( $25.6 \mathrm{~ms}$ default). Time-integrated values are sent over 100 Mbps Ethernet to a LabView program that estimates the spectral kurtosis of all 2048 channels for each integration time interval. Spectral kurtosis is the fourth-order moment of the magnitude of the short-time Fourier transform. It can be used in data normality tests (astronomic data is usually Gaussian). If skewness is available, too, the Jacque-Bera test can be applied.

The simplicity of the spectral kurtosis approach prompted us to investigate software possibilities. In addition, there is an interesting application of RFI detection algorithms to detect planetary lightning in the solar system [4].

Three methods can be used for one-pass estimation of sample or channel probability distribution central moments. The first method is the fast but numerically unstable sum of powers method used in the KSRBL. The second method is similar but numerically stable set of pair-wise update formulas summarized in the Sandia Labs report [5]. The third method is to delay computing the central moments and instead build an on-line histogram of the samples, from which all central moments (and different entropies) can be extracted after the desired number of samples have been binned. For kurtosis spectra, kurtosis is computed on time-domain data windows. On the other hand, for spectral kurtosis the methods are applied to frequency domain data, e.g. a post-DFT bin time series or the output of a wavelet transform.

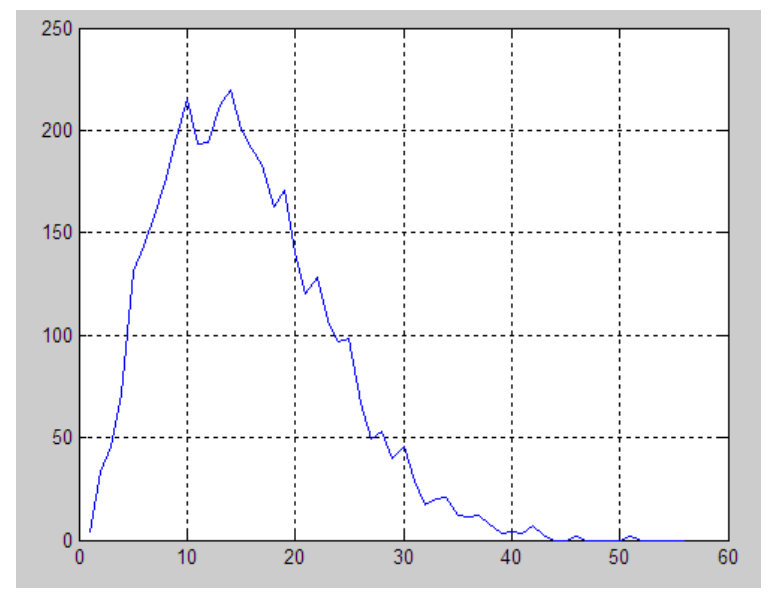

Figure 3: Histogram of the time series of magnitude values from one FFT bin. Mean, variance, skewness, kurtosis, entropy estimates can be extracted directly after binning the last sample of the integration interval.

The third method (histogramming) was implemented in a new software tool (kurtosistoy) derived from MRO high-resolution spectrometer and spacecraft tracking software [6]. Kurtosistoy can process common VLBI and several raw data formats. Spectral resolution $\left(N_{D F T}\right.$-point DFT discrete fourier transform) and integration times are configurable. Each of the $N_{D F T} / 2$ channels is binned into its own histogram. Histogram length is $2 N_{D F T}+1$ and DFT magnitude values are scaled by $1 /\left(2 N_{\text {bits }}{ }^{-1}\right)$ into histogram bin indices.

With 2-bit and 8-bit raw input data, throughput is $90 \mathrm{Ms} / \mathrm{s}$ per CPU core. With data in Mark5A format read via the mark5access library (NRAO), throughput per core drops to $30 \mathrm{Ms} / \mathrm{s}$. The method is memory and cache but not arithmetic intensive. 
Kurtosistoy writes spectral skewness, kurtosis and entropy estimates of each time integration interval. The output files are later analyzed in a set of Matlab scripts to apply entropy methods, the Jacque-Bera test with skewness and kurtosis, or simply a kurtosis threshold normality criterion.

Data from a three-station ESA Mars Express (MEX) observation on 03.03.2010 with Wettzell, Yebes, Metsähovi observing X-band was analyzed at different time scales in the kurtosistoy software to find the presence of natural RFI from Mars lightning. Software was able to catch the MEX carrier and ranging tones. However no RFI was present during the two hours of MEX observation. We hope to evaluate future observations of MEX for potential lightning detection.

\section{Conclusions}

Being aware of the RFI environment is very important in the field of radio astronomy because of the extremely tiny signal power levels employed. Concerning the overall RFI levels Metsähovi benefits from its rather remote location and the high frequency bands utilised for observations. Currently the RFI situation is handled by passive monitoring of the receivers IF band frequencies. RFI mitigation methods currently in use are time blanking and RFI-free frequency selection. With the scanning method the overall spectrum cleanness can be investigated and verified easily, but occasional short-period interferences will most probably remain undetected. Contaminated data is removed by hand when needed.

The relations to the Finnish Communications Regulatory Authority have traditionally been good, which enables easier mitigation of existing and future threats.

There are future plans of doing RFI monitoring and excision by software. For example, different kurtosis methods have recently been developed and excercised. There are also plans to incorporate some kind of AI into the current frequency scanning system. The system could hunt and recognize some kinds of RFI signals, and start to track and record them if detected.

\section{References}

[1] Engelberg J., Mujunen A., Oinaskallio E., Radio Spectrum Monitoring System - spektrd, Metsähovi Reports, HUT-KURP-16, Espoo 2000

[2] Berkeley University, Center for Astronomy Signal Processing and Electronics Research, iBOB FPGA board hardware platform, http://casper.berkeley.edu/wiki/IBOB

[3] Zhiwei Liu, Korean Solar Radio Burst Locator, Spectral Kurtosis (SK) Spectrometer, http://casper.berkeley.edu/wiki/Kurtosis_Spectrometer 2009

[4] Ruf et al., Emission of non-thermal microwave radiation by a Martian dust storm, AGU, Geophysical Research Letters, 36 (13), 16th July 2009

[5] Philippe Pébay, Formulas for Robust, One-Pass Parallel Computation of Covariances and Arbitrary-Order Statistical Moments, Technical Report SAND2008-6212, Sandia National Laboratories, http://infoserve.sandia.gov/sand_doc/2008/086212.pdf 2008

[6] J. Wagner, S. Pogrebenko, G. Molera et al, Evaluation of new high-performance spectrometer and spacecraft tracking software, 8th RadioNet Engineering Forum Workshop, Yebes, http://www.radionet-eu.org/rnwiki/UpgradingtheHorizonPresentations/, 24-25 November 2008 